\title{
The effect of prior reward magnitude on the successive negative contrast effect
}

\author{
EARL R. McHEWITT \\ Southern Illinois University, Carbondale, Illinois 62901
}

\begin{abstract}
Separate groups $(N=10)$ of albino rats received nondifferential large $(L)$, nondifferential small (S), or no reward training prior to differential reward training (Groups L-LS, S-LS, and LS, respectively) in a nonchoice brightness discrimination apparatus. A fourth condition received small reward in each discriminandum throughout training. Results suggested that the extent of simultaneous negative contrast was greater for Group L-LS than for Group S-LS and clearly indicated that the development of discrimination and negative contrast effects was retarded for the prior reward conditions relative to the standard differential conditioning group (LS).
\end{abstract}

In differential conditioning, subjects receiving contrasted large and small reward display slower speeds to small reward than do subjects receiving small reward in both discriminanda (cf. Dunham, 1968; McHose, 1970). The depressed performance in the small reward alley has been termed a simultaneous negative contrast effect (NCE).

The present concern is with the effect of prior reward training on the simultaneous NCE. Previous studies (e.g., Platt \& Gay, 1969; Spear \& Spitzner, 1969) consistently report greater simultaneous NCEs following nondifferential experience with large as compared to small reward. There are differences however in the interpretation of this effect. One interpretation, consistent with frustration-based accounts of contrast phenomena (cf. Black, 1968; Bower, 1961), views the prior large reward experience as actively facilitating the development of simultaneous contrast (Platt \& Gay, 1969). Another view focuses on the effect of the small reward training. Spear and Spitzner (1969) employing a design similar to Platt and Gay's (1969), but a choice procedure, also found a greater simultaneous NCE following large reward, but the magnitude of the NCE was comparable to that of a standard differential conditioning group with no prior reward training. The conclusion was that prior small reward training retarded the development of the NCE, that subjects experiencing small reward were somehow protected against otherwise depressed performance for small reward in the differential conditioning phase.

The present study is essentially a replication of the Platt and Gay (1969) study with the addition of a standard differential conditioning group. It also provides further data relevant to determining the nature of the effect of prior reward training on the simultaneous NCE.

This research was supported in part by Grant MH 10340 to James H. McHose, Southern Illinois University, who sponsors and takes full editorial responsibility for this paper. Requests for reprints should be sent to Earl $R$. McHewitt, who is now at the Department of Psychology, King College, Bristol, Tennessee 37620.

\section{METHOD}

Forty naive albino rats of the Holtzman strain, approximately 120 days old at the beginning of the experiment. served as subjects.

The apparatus consisted of a $25-\mathrm{cm}$ midgray startbox and two $96-\mathrm{cm}$ alleys; both alleys, which included $291 / 2-\mathrm{cm}$ goal sections, could be aligned with the startbox. One alley (including goal section) was painted flat black, the other flat white. For all sections, the inner height was $10 \mathrm{~cm}$, and the inner width, $9 \mathrm{~cm}$. A translucent Plexiglas covered all units of the apparatus. An opaque solenoid-operated door separated the startbox from the alleys. Clear Plexiglas guillotine-type retrace doors separated the goal and runway sections of each alley. Photocell-clock circuitry provided traversal times over the first $90 \mathrm{~cm}$, including a $30-\mathrm{cm}$ start-time measure.

Approximately 9 day's prior to the first training day, Day 10 , subjects were placed on a 23 -h food deprivation/free water access schedule which was maintained throughout the experiment. On Days 8-9, subjects were allowed to explore the start and run sections of the apparatus, and, upon the return to the home cages, subjects were fed approximately $.5 \mathrm{~g}$ of $45-\mathrm{mg}$ Noyes pellets.

The 40 subjects were randomly assigned to four groups, Groups L-LS, S-LS, LS and SS. The groups are designated according to the amount of reinforcement received in the S+ alley (e.g., black) and $\mathrm{S}-$ (e.g., white) during the pre- and postshift periods. For example, Group L-LS received 12 (L) $45-\mathrm{mg}$ pellets in both alleys during preshift and 12 pellets in S+ and 1 pellet $(S)$ in $S-$ in the postshift period. The nonshifted conditions are similarly designated; Group SS, for example, received one pellet in both $S+$ and $S-$ throughout both phases. All subjects received four trials/day according to the following recursive cycle of black $(B)$ and white $(W)$ trials: $B W B W, W W B B$, BBWW, WBWB, BWWB for a total of 144 trials, 72 in each period.

Subjects were run in squads with the restriction that both $\mathrm{S}+$ brightness conditions be present in any squad. Each subject in a squad received an initial trial before any subject received the second trial, etc. The intertrial interval was approximately $6 \mathrm{~min}$. On each trial, the start door was opened after the subject had oriented toward the door for $3 \mathrm{sec}$, and the subject was removed immediately after reward consumption.

\section{RESULTS AND DISCUSSION}

Group mean start speeds for the first 4 and the last 4 days of the postshift period are presented in Figure 1. 


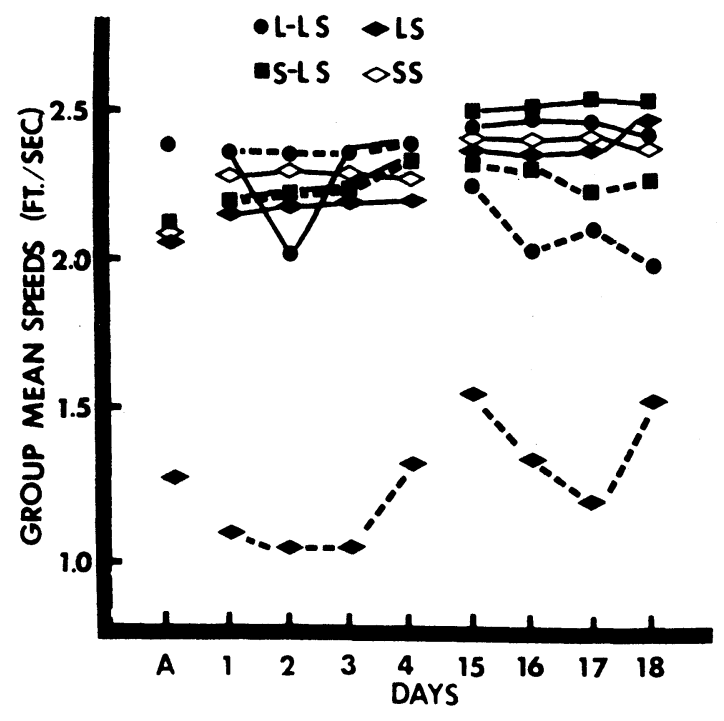

Figure 1. Terminal preshift and postshift start speeds for the various conditions as a function of days.

Block A represents the last 2 days of preshift training. "S+" and "S-" trials did not differ for Group SS and are represented by a single trend.

As may be seen at Block A, the pattern of preshift data is typical of previous differential conditioning data (cf. McHose, 1970). The effect of reward magnitude was significant both within and between subjects, S+ vs. Sspeeds for Group LS $(p<.01)$ and faster speeds for Group L-LS relative to those of Groups S-LS and SS $(p<.05)$. Both $S+$ and $S-$ speeds for Group LS were depressed $(p<.01)$ relative to those of the nondifferential large and small reward conditions, respectively. Thus, typical reward magnitude and simultaneous contrast effects were present in the preshift period.

An inspection of the early postshift speeds in Figure 1 indicates comparable performance levels for the various conditions with the exception of the continued slower speeds in St and S- for Group LS and the drop in S+ speeds for Group L-LS at Day 2. An analysis of variance performed over Days $2-3$ yielded a significant $(\mathrm{p}<.01)$ groups by discriminanda (S+ vs. S- speeds) effect, $\mathrm{F}(3,32)=9.81$. Pair comparisons (Tukey a) indicated that only the $\mathrm{S}-$ speeds for Group LS were reliably $(p<.01)$ slower than those for the small reward control, Group SS. A comparison ( $t$ test) of S+ speeds for Groups L-LS and SS at Day 2 was not significant. It should be noted that an inspection at Days 2 and 3 of both S+ and S- speeds for Group L-LS following large reward (S+ trials) vs. those following small reward (S- trials) did not reveal any consistent differences. Thus, successive negative contrast, frequently observed in simple instrumental conditioning (cf. Capaldi \& Lynch, 1967; Dunham, 1968) following a shift from large to small reward, did not obtain for Group L-LS.

The finding that the postshift speeds to small reward for Group L-LS were not depressed relative to those of Group SS is consistent with a previous report (McHewitt, 1974) of no successive negative contrast, in either discriminandum, for such a condition when compared to a postshift control receiving nondifferentiated large and small reward. It would seem then that the early postshift performance, before differential responding, for conditions like Group L-LS may be best described if the manipulation is viewed as a shift from large to varied reward and not as involving a situation-specific incentive reduction, i.e., a shift from large to small reward in a single alley. Viewed in this manner, the present pre- to postshift reward reduction may not have resulted in a sufficient incentive difference to produce the successive NCE (Peters \& McHose, 1974)

Turning now to the data of primary interest, it may be seen at Day 18 that while each of the differentially rewarded conditions displayed faster speeds to $S+$ than to $\mathrm{S}-$, the S+ vs. S- speed difference was largest for Group LS. Also, this difference was larger for Group L-LS than for Group S-LS. Comparing S- speeds of these conditions with those of Group SS results in a similar order, S- speeds of Group LS being the slowest. That is, Group LS showed the largest amount of simultaneous negative contrast. An analysis of variance of St and S- speeds over Days 17-18, including groups and $\mathrm{S}+$ brightness as factors, yielded a significant $(\mathrm{p}<.01)$ Groups by $\mathrm{S}+$ vs. $\mathrm{S}-$ speeds interaction, $\mathrm{F}(3,32)=6.47$. Comparisons ( $\mathrm{t}$ tests) of $\mathrm{S}+$ vs. $\mathrm{S}-$ speeds were significant $(p<.01)$ for Groups $L S$ and L-LS, but not, conventionally, for Group S-LS $(p<.10)$. Subsequent comparisons (Tukey a) of $S-$ speeds for these conditions relative to those of Group SS were as follows: Group LS $(p<.01)$, Group L-LS $(p<.10)$, and for Group S-LS $(p>.10)$. Additional comparisons of relative $\mathrm{S}+$ and $\mathrm{S}$ - speeds were not significant except for the slower S- speeds of Group LS relative to those of Groups L-LS and S-LS $(\mathrm{p}<.01)$.

While the suggestion of slower $S-$ speeds for Group L-LS relative to those of Group S-LS is in agreement with previous reports (Platt \& Gay, 1969; Spear \& Spitzner, 1969), the relative performance of these same conditions to that of the standard differential reward condition is totally unexpected. In direct comparison with Group LS, the development of discrimination and simultaneous NCEs was markedly retarded for the conditions receiving prior reward training, this being the case even though these conditions received as many differentially rewarded trials as did Group LS in the preshift period. The overall pattern of results, then, questions previous interpretations. Specifically, the notion (Platt \& Gay, 1969) that prior large reward in $\mathrm{S}$ - should maximize the discrepancy between obtained and expected rewards, producing maximum inhibition in S- for Group L-LS during differential conditioning, is not supported by the present data.

Similarly, the view (Spear \& Spitzner, 1969) that 
experience with small reward lessens the disruptive effects of the simultaneous contrast manipulation finds only partial support in these data, as prior large reward also attenuated the development of negative contrast. Thus. while these views expect the direct relationship between the extent of simultaneous negative contrast and prior reward magnitude. none would seem to predict the markedly retarded development of differential responding for the same conditions.

\section{REFERENCES}

Black, R. W. Shifts in magnitude of reward and contrast effects in instrumental and selective learning: A reinterpretation Psychological Reriew, 1968, 75, 114-126.

Psycholion conditioning. Journal of Experimental Psy chology, 1961,62,196-199.

apaldi E. G., \& Lynch, D. Repeated shifts in reward manitude: Evidence in favor of an associational and absolute (noncontextual) interpretation. Journal of Experimental Psy chology, 1967, 75, 226-235.
Dunham, P. J. Contrasted conditions of reinforcement: A selective critique. Psychological Bulletin, 1968,69, 295-315. M cHewitt, E. R. Reward shift effects in differential conditioning. Journal of Experimental Psy chology, 1974, 103, 646-651.

McHose, J. H. Relative reinforcement effects: $S_{1} / S_{2}$ and $S_{1} / S_{1}$ paradigms in instrumental conditioning. Psychological Review, $1970,77,135-146$.

Peters, D. P.. \& McHose, J. H. Effects of varied preshift reward magnitude on successive negative contrast in rats. Journal of Comparative and Physiological Psychology, 1974, 86, 85-95.

Platt, J. R.. \& Gay, R. A. Differential magnitude of reward conditioning as a function of predifferential reward magnitude. Journal of Experimental Psychology, 1968, 77, 393-396.

Spear, N. E., \& Spitzner, J. H. Influence of degree of training and prior reinforcer magnitude on contrast effects and resistance to extinction within S. Journal of Comparative and Phy siological Psychology, 1969, 68, 427-433.

(Received for publication April 2, 1975.) 